\title{
Toward Customized Hybrid Fuel-Cell and Battery-powered Mobile Device for Individual Users
}

\author{
KAIGE YAN and JINGWEIJIA TAN, Jilin University, China \\ LONGJUN LIU, Xi'an Jiaotong University, China \\ XINGYAO ZHANG, STANKO R. BRANKOVIC, JINGHONG CHEN, and XIN FU, \\ University of Houston, USA
}

\begin{abstract}
Rapidly evolving technologies and applications of mobile devices inevitably increase the power demands on the battery. However, the development of batteries can hardly keep pace with the fast-growing demands, leading to short battery life, which becomes the top complaints from customers. In this article, we investigate a novel energy supply technology, fuel cell (FC), and leverage its advantages of providing long-term energy storage to build a hybrid FC-battery power system. Therefore, mobile device operation time is dramatically extended, and users are no longer bothered by battery recharging. We examine real-world smartphone usage data and find that a naive hybrid power system cannot meet many users' highly diversified power demands. We thus propose an OS-level power management policy that reduces the device power consumption for each power peak to solve this mismatch. This technique trades the quality-of-service $(\mathrm{QoS})$ for a larger FC ratio in the system and thus much longer device operation time. We further observe that the user's personality largely determines his/her satisfaction with the QoS degradation and the operation time extension. Thus, applying a hybrid system with fixed configuration (i.e., peak throttling level coupled with corresponding FC/battery ratio) fails to satisfy every user. We then explore customized hybrid system configuration based on each individual user's personality to deliver the optimal satisfaction for him/her. The experimental results show that our personality-aware hybrid FC-battery solution can achieve $4 \times$ longer operation time and $25 \%$ higher satisfaction score compared to the common setting for state-of-the-art mobile devices.
\end{abstract}

\section{CCS Concepts: • Hardware $\rightarrow$ Batteries; Fuel-based energy; $\bullet$ Human-centered computing $\rightarrow$ User} models;

Additional Key Words and Phrases: Mobile devices, fuel cell, battery, customize

\footnotetext{
The work is supported in part by National Natural Science Foundation of China (NSFC) under Grant No. 61902142, No. 61802143, Jilin Scientific and Technological Development Program under Grant No. 20190201189JC, No. 20180101046JC, 20190701016GH, and Research Project by the Education Department of Jilin Province under Grant No. JJKH20190159KJ. This research is also partially supported by U.S. National Science Foundation grants CCF-1619243, CCF-1537085(CAREER), CCF-1900904.

Authors' addresses: K. Yan (corresponding author), Jilin University, College of Communication Engineering, 2699 Qianjin Street, Changchun, 130012, China; email: yankaige@jlu.edu.cn; J. Tan, Jilin University, College of Computer Science and Technology, 2699 Qianjin Street, Changchun, 130012, China; L. Liu, Xi'an Jiaotong University, College of Artificial Intelligence, Xi'an, 710049, China; X. Zhang, S. R. Brankovic, J. Chen, and X. Fu, University of Houston, Department of Electrical and Computer Engineering, Houston, TX 77004.

Permission to make digital or hard copies of all or part of this work for personal or classroom use is granted without fee provided that copies are not made or distributed for profit or commercial advantage and that copies bear this notice and the full citation on the first page. Copyrights for components of this work owned by others than the author(s) must be honored. Abstracting with credit is permitted. To copy otherwise, or republish, to post on servers or to redistribute to lists, requires prior specific permission and/or a fee. Request permissions from permissions@acm.org.

(C) 2019 Copyright held by the owner/author(s). Publication rights licensed to ACM. 1539-9087/2019/11-ART123 \$15.00

https://doi.org/10.1145/3362033
} 


\section{ACM Reference format:}

Kaige Yan, Jingweijia Tan, Longjun Liu, Xingyao Zhang, Stanko R. Brankovic, Jinghong Chen, and Xin Fu. 2019. Toward Customized Hybrid Fuel-Cell and Battery-powered Mobile Device for Individual Users. ACM Trans. Embed. Comput. Syst. 18, 6, Article 123 (November 2019), 20 pages.

https://doi.org/10.1145/3362033

\section{INTRODUCTION}

Today's mobile device technology are evolveing rapidly, and more cutting edge technologies (e.g., the augmented reality, voice control, hologram) are being introduced to the devices, which inevitably increases the power demands for the battery. Moreover, the mobile device producers are pushing toward lighter and thinner devices to improve usability, which makes the battery design even more challenging, since it needs to provide a large amount of energy under very strict constraints. Unfortunately, the state-of-the-art battery for mobile devices, i.e., the Li-ion batteries, could not keep up the pace, and battery life is usually sacrificed to meet those increasing power demands and design constraints. As a result, the battery life becomes one of the top complaints about modern mobile devices. An ideal energy supply system should be able to provide the long-term energy storage for the mobile devices and meanwhile still meet the high power demands.

Besides Li-ion batteries, there have been various alternative energy supply technologies. Among them, super capacitors (SCs) and fuel cells (FCs) are generally considered as good candidates for energy storage in computer systems. Figure 1 compares the specific energy (in $\mathrm{Wh} / \mathrm{kg}$ ) and specific power (in watts per kilogram) of SCs, Li-ion batteries, and FCs. Specific energy denotes the amount of energy provided by a unit of weight, and specific power denotes the maximal power supported by a unit of weight. As shown, SCs have the highest specific power (two order-of-magnitude higher than the Li-ion batteries) but very low specific energy (one to two order-of-magnitude lower than the Li-ion batteries). Due to their extremely low specific energy, SCs can only provide short-term energy storage. Meanwhile, their high specific power is far beyond the power demands of mobile devices. Thus, SCs are not suitable for mobile devices. Instead, they are promising to be used in cases where burst-mode power delivery is needed, e.g., data centers [33]. FCs have one- to two-orders-of-magnitude-higher specific energy than the Li-ion batteries, but their specific power is slightly lower. Because of the obvious advantage of high specific energy (i.e., the long-term energy storage), FCs have been viewed as one of the most promising candidate for mobile devices. However, FCs also suffer some major disadvantages, i.e., low specific power and slow dynamic responses, which must be taken into consideration when using them as the energy source for mobile devices.

It is well known that mobile device are not active and running applications all the time; their power demands remain low during the idle state and will experience a rapid increase when there is a user action. Leveraging this unique characteristic, in this work, we propose the hybrid FC and battery design to power the emerging mobile platforms. FCs will provide the basic power and batteries are used to compensate the slow dynamic response of FCs and satisfy high power demand. FC and battery hybrid system has been widely studied before for portable device, electric cars, unmanned aerial vehicles, and so on [16, 31, 39]. However, how to design the optimal hybrid energy solution for each individual mobile user based on his/her usage still needs to be investigated.

We then investigate the real-world smartphone usage data and observe the highly diversified user power demands due to distinct user behaviors. A naive hybrid power system design with either huge FCs or large battery fails to meet many users' power requirements. We then explore an OS-level power management policy that reduces the device's peak power consumption by $\alpha \%$ 


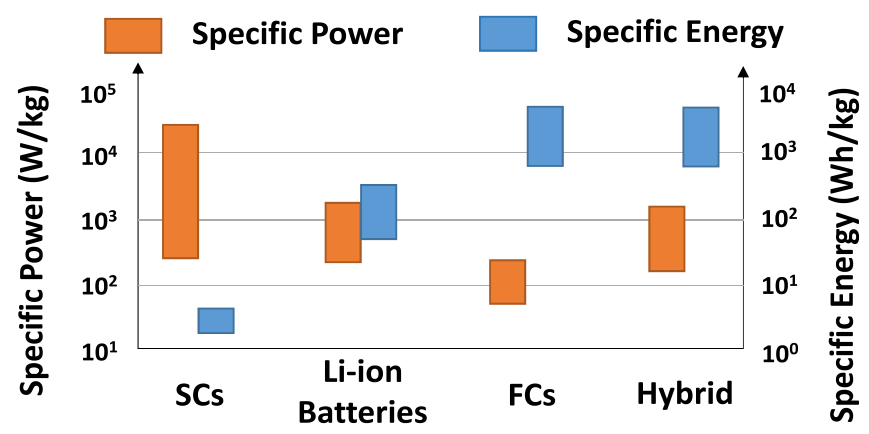

Fig. 1. The specific energy and specific power of super capacitor (SCs), Li-ion batteries, and fuel cell (FCs).

to solve those mismatches between the power supply in the hybrid system and the power demands. With a large throttling level, high power demand is reduced and small battery size in the hybrid system suffices. Therefore, there is a one-to-one mapping between peak throttling level and $\mathrm{FC} /$ battery ratio. We define the pair of $\langle\alpha \%, \eta \%\rangle$ as the configuration of our hybrid system, where $\alpha \%$ specifies the peak throttling level and $\eta \%$ indicates the portion of battery in the hybrid system. Obviously, a high peak throttling level $\alpha \%$ will cause more quality-of-service (QoS) degradation. However, since $\eta \%$ can also be decreased accordingly, and, thus, there is more room for FC and the operation time are extended. As a key observation in this study, we find that the user's personality largely determines his/her perception on the QoS degradation and the long operation time brought by our novel power system. For example, people exhibiting strong trait on neuroticism can tolerate the high QoS loss caused by an aggressive peak throttling technique, as they have relatively light device usage thus enjoy its benefit on the long device operation time. To the contrary, the conscientious people prefer the high QoS, since they are highly organized and usually have regular charging behavior, and, thus, the long operation time is less attractive to them. As can be seen, adopting a hybrid system with fixed configuration for all users fails to consider their personality and thus cannot deliver the optimal user experience.

In this study, we explore a customized hybrid FC and battery system configuration for each individual user according to his/her personality, therefore achieving optimal user satisfaction. We conduct a comprehensive survey on 60 users and build a linear regression model to find out the optimal peak throttling level given a user's personality. Evaluated with the real-world mobile user data, we find that our customized hybrid FC and battery power system based on each individual user's personality is able to achieve $4 \times$ longer operation time and the overall satisfaction score is improved by $25 \%$ comparing with the baseline case of using the LCO battery to power the device on average across all investigated users.

To summarize, this article makes the following contributions:

- We propose and implement a hybrid fuel cell and battery power system for emerging mobile devices.

- We propose an OS-level power management policy that reduces the peak power consumption to solve the mismatch between the power supply in our hybrid power system and mobile users' power demands.

- We further explore customized hybrid FC and battery system configuration for each individual user according to his/her personality, which achieves the optimal user satisfaction per user. 


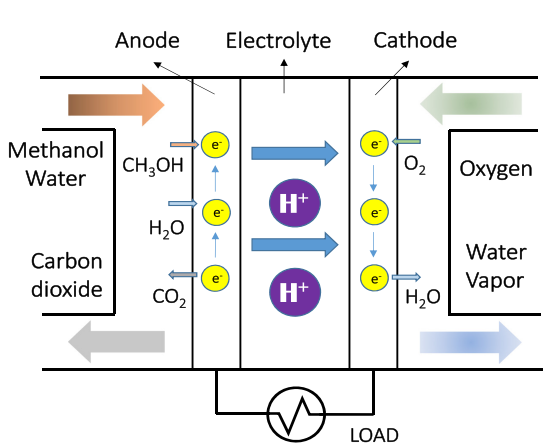

(a)

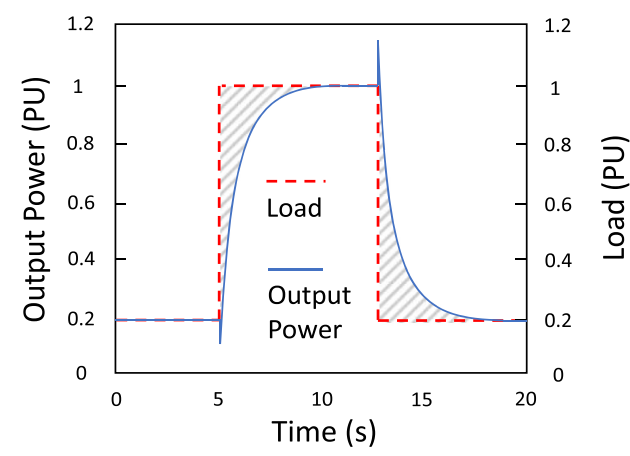

(b)

Fig. 2. The characteristics of DMFC.

\section{BACKGROUND}

\subsection{Fuel Cells}

A fuel cell is a device that can convert the chemical energy in the fuel to electricity. It has been recognized as one of the most promising energy sources in the 21st century and is widely used in many fields, including power stations, vehicles, un-interruptible power supplies (UPS), and so on, due to its high specific energy $[12,13,59]$. There are various types of fuel cells, such as the direct methanol fuel cell (DMFC), the hydrogen-based polymer electrolyte membrane fuel cell (PEMFC), and so on. DMFC is considered more suitable for mobile devices, as it not only can operate at room temperature but also uses methanol as the fuel, which is easy to store and transport without additional fuel reforming process like hydrogen-based PEMFC [25]. The DMFC has very high specific energy, up to $\sim 5000 \mathrm{Wh} / \mathrm{kg}$, compared with $\sim 150 \mathrm{Wh} / \mathrm{kg}$ for the common Li-ion batteries used in modern mobile devices [5, 10, 62]. It contains two electrodes, i.e., anode and cathode, which are separated by the electrolyte, as shown in Figure 2(a). The following chemical reactions occur at the anode and cathode of DMFC, respectively:

$$
\begin{aligned}
\mathrm{CH}_{3} \mathrm{OH}+\mathrm{H}_{2} \mathrm{O} & \rightarrow 6 \mathrm{H}^{+}+6 e^{-}+\mathrm{CO}_{2} \quad \text { (anode) } \\
\frac{3}{2} \mathrm{O}_{2}+6 \mathrm{H}^{+}+6 e^{-} & \rightarrow 3 \mathrm{H}_{2} \mathrm{O} \quad \text { (cathode) } .
\end{aligned}
$$

Fuel cells have some special characteristics, which must be taken into consideration when using them as the energy source for mobile devices. To analyze and illustrate these characteristics, we simulate a passive air-breathing DMFC using the analytical model developed in Reference [42]. Fuel cells can stack multiple layers of flow beds. Common designs of flow beds include serpentine, parallel, interdigitated, and pin [27, 30, 32]. For micro DMFC, the single-serpentine flow bed design is considered to be the most favorable [53]. To make the size of DMFC comparable to the smartphone battery, we consider such design with total active area of $16 \mathrm{~cm}^{2}$. Under room temperature, its efficiency is $\sim 20 \%$, and, thus, its effective specific energy is $\sim 1000 \mathrm{Wh} / \mathrm{kg}$, which is 6X that of common Li-ion batteries. For FCs, there exists a Maximum Power Point (MPP). With current density smaller than the MPP, the output power grows when the current becomes larger. If the current density is beyond the MPP, then the output power will drop sharply. Maximum Power Point Tracking (MPPT) is usually adopted to pull the most power out of FCs $[18,26]$. The dynamic performance of our DMFC is shown in Figure 2(b). It illustrates the simulated voltage curve with current load-up and load-down (the baseline for per unit output power is $1 \mathrm{~W}$ ). FCs are sluggish in responding to load changes. It fails to provide enough power in the next few seconds after sharp 
load-up, while still generates excessive power after load-down. The voltage needs around $5 \mathrm{~s}$ to keep up and stabilize, which is clearly to slow for CPUs. The reason for this phenomenon is that many dynamic processes, e.g., gas diffusion effects, liquid water transportation and flooding, and so on, take place during the load-up and load-down, and these processes take seconds to finish [57]. Besides the slow dynamic response, FCs usually fail to provide enough power for mobile devices. Our DMFC has around $1 \mathrm{~W}$ of maximal output power; however, the power traces of real smartphone users suggest that the power consumption of mobile devices can be up tp $\sim 4 \mathrm{~W}$ [48].

\subsection{Batteries}

Batteries, as the most commonly used energy supply for today's mobile devices, are devices that can convert the stored chemical energy into electricity and vice versa. The former process is called battery discharging and the latter is charging. The current level during charging (discharging) is usually called the C-rate. For example, a 5C charging (discharging) rate will replenish (deplete) the battery in $1 / 5$ hour. The endurance of battery can be measured by the battery lifecycle, i.e., the number of complete charge/discharge cycles that a battery can use. Lithium-ion batteries are widely used for portable devices because of their relatively high specific energy ( $\mathrm{Li}$ is the lightest metal) and great stability, comparing with other types of batteries, e.g., lead acid. Even lithium-ion batteries have different types, e.g., lithium cobalt oxide $\left(\mathrm{LiCoO}_{2}\right.$ or $\left.\mathrm{LCO}\right)$, lithium titanate $\left(\mathrm{Li}_{4} \mathrm{Ti}_{5} \mathrm{O}_{12}\right.$ or LTO), and so on. The LCO battery is the most popular choice for smartphones because of its low cost. It has high specific energy $(\sim 150 \mathrm{Wh} / \mathrm{kg})$ but low discharging rate (1C) and low lifecycle $(\sim 750)$ [5, 44]. Today's smartphones can equip around $3500 \mathrm{mAh}$ LCO battery [15]. Still, it can only last for around one day under normal usage. The LTO battery has low specific energy $(\sim 75 \mathrm{Wh} / \mathrm{kg})$ but high discharging rate $(30 \mathrm{C})$, long lifecycle ( 5000$)$, and also around $98 \%$ re-charge efficiency $[3,44]$. The LTO battery is seldom used for smartphones due to its low specific energy; however, it becomes more useful when high charging rate and long endurance are needed.

\subsection{The Diversified Power Demands of Mobile Users}

To design an energy source for mobile devices, real-world smartphone usage must be examined. It has been found that different mobile users have highly diversified application usage [17]. C. Shepard et al. [48] presented LiveLab traces, which recorded iPhone usage data of 24 Rice University students from February 2010 to February 2011 and 10 Houston Community College students from September 2010 to February 2011, including the application usage, power, charging, WiFi connection, Web surfing history, and so on. We investigate the power traces of the 15 most popular applications for all users in the LiveLab data and find diversified power demands of these applications, e.g., the camera consumes $\sim 1.8 \mathrm{~W}$ while the weather application only consumes $\sim 0.8$ $\mathrm{W}$ on average cross all users. Even for the same application, its power varies substantially for different users. For example, the power for Pandora ranges from 0.4 to $1.8 \mathrm{~W}$.

Figure 3 further presents the distribution of device power for each user in the LiveLab data. As shown, the percentage that device power exceeds $2 \mathrm{~W}$ is low $(<10 \%)$ for all users, although modern smartphone's peak power can achieve $\sim 4 \mathrm{~W}$. This suggests that the high output power capability of batteries is not always needed. Then, we focus on the distribution of the power in the range $[0 \mathrm{~W}, 2 \mathrm{~W}]$ and it is obvious that users have totally different power profiles, as shown in the figure. Some users (e.g., B00, D07) have quite light usage with their smartphones running at super low power mode $(<0.2 \mathrm{~W})$ almost half of the operation time, and their smartphones remain idle for up to $70 \%$ of the time (when the smartphone is idle, its power is generally around $0.5 \mathrm{~W}$ or less). To the contrary, some users (e.g., A09, D05) heavily use their smartphones, and the idle time only accounts for $25 \%$ of the total operation time. 


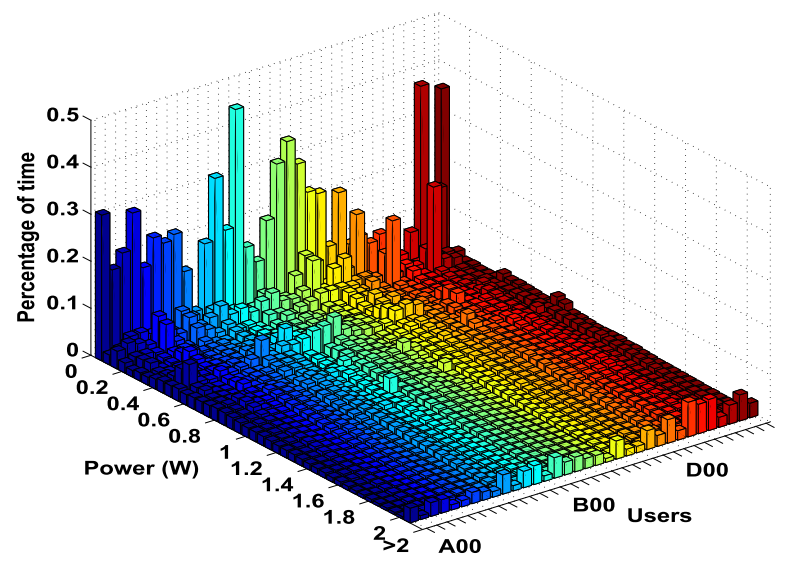

Fig. 3. The distribution of device power for all users in the LiveLab trace. On the $x$-axis, the users are ranked as A00-A12, B00-B11, and D00-D09 from left to right (different colors are for easy reading and have no specific meaning).

\section{DESIGN OF HYBRID FUEL CELL AND BATTERY POWER SYSTEM FOR MOBILE DEVICES}

In this section, we first introduce the architecture of hybrid FC and battery power system. To enable such a hybrid system for mobile devices, we also design an OS-level power management policy. Then, we further quantitatively study the relationship between the configurations of the hybrid system and the power management policy.

\subsection{The Architecture of the Hybrid Power System}

Although FCs have remarkably high specific energy, their slow dynamic response and low specific power make them unsuitable to be the sole energy source for mobile devices. However, Li-ion batteries, especially LTO batteries, can support high C-rate (i.e., high specific power) and long endurance, which can offset the disadvantages of FCs. Thus, we explore a hybrid FC and battery power system for the mobile devices, which leverages the advantages and combats the disadvantages of these two power supplies to substantially extend the device operation time, hence, greatly improving the user experience.

The architecture of our hybrid FC and battery power system is shown in Figure 4. In this hybrid system, the LTO battery, whose specific energy is roughly $1 / 2$ of the LCO battery, will collaborate with our passive air-breathing DMFC as discussed in Section 2.1, which has $1 \mathrm{~W}$ of maximum power and $6 \times$ specific energy comparing with LCO batteries. Without explicitly mentioning, batteries in the following sections means the LTO batteries. The output voltage of a single DMFC cell is only around $0.4-0.8 \mathrm{~V}$, and converting such low voltage to the required voltage of mobile devices can yield high loss $[44,62]$. Thus, four DMFC cells are stacked in sequence, which can provide 0.8 to $2.4 \mathrm{~V}$ and improves the converting efficiency. Battery charging is controlled by switch $Q_{1}$, and the top converter is used to convert the FC stack output voltage to battery charging voltage. Switches $Q_{3}$ and $Q_{2}$, together with the other two converters, are responsible for enabling FC stack and battery output and transforming their voltages to the required load voltage, respectively.

The core of our hybrid system is the Energy Management System (EMS). It takes the device power $P_{\text {load }}$ and battery State-of-Charge (SoC) as inputs. Based on the power management strategies, EMS generates the reference currents. Then proportional-integral (PI) controllers and Pulse 


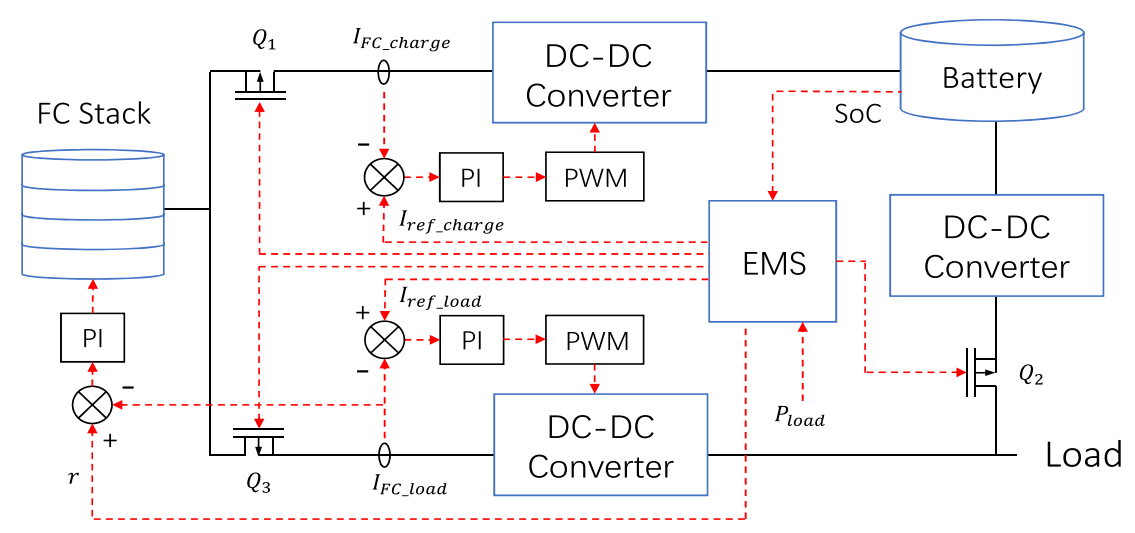

Fig. 4. The overview of the hybrid FC and battery power system.

Width Modulations (PWMs) are used to determine the duty cycle of converters. The detailed states and corresponding management strategies are as follows.

State 1: Load power $P_{l o a d}$ is higher than the maximal power of FC stack $P_{F C_{-} M a x}$ and SoC of battery is higher than the lowest operating threshold, i.e., battery is not empty. In this case, the battery will be responsible for compensating the slow dynamic response of FC and satisfying high power demand. $Q_{1}$ is off, while $Q_{2}$ and $Q_{3}$ is on. EMS adjusts the water to methanol molar ratio $r$ to a value $R_{\text {Max }}$, such that FC outputs the maximal power. It also sets the reference current $I_{\text {ref_load }}$, which corresponds to the MPP.

State 2: Load power $P_{l o a d}$ is lower than the maximal power of FC $P_{F C \_M A X}$, and the battery is not full. In this case, the FC will provide the load power and charge the battery in the meantime. $Q_{1}$ and $Q_{3}$ is on, while $Q_{2}$ is off. EMS sets $r=R_{\text {Max }}$. It also sets $I_{\text {ref_load }}$, which corresponds to the $P_{\text {load }}$, and $I_{\text {ref_charge }}$, which corresponds to the $P_{F C_{-} \text {Max }}-P_{\text {load }}$.

State 3: The power of load $P_{\text {load }}$ is lower than the maximal power of FC $P_{F C}$ MAX and the battery is full. In this case, the battery will provide the load power. $Q_{1}$ and $Q_{3}$ are both off, while $Q_{2}$ is on. The SoC of battery need to be reduced to certain threshold, such that the battery can take the excessive power of FC during load-down.

State 4: The battery is empty. In this case, the FC will provide the load power and charge the battery in the meantime. $Q_{1}$ and $Q_{3}$ are on, while $Q_{2}$ is off, $r=R_{\text {Max }}$, and EMS also sets $I_{\text {ref_load }}$, which corresponds to the $P_{\text {load }}$. The device is forced to operated at idle state and the change of state is prohibited.

\subsection{OS-Level Power Throttling Policy}

The hybrid system can ensure normal functioning of the device except in state four when the battery is empty. For users with heavy power demand, a hybrid system with insufficient battery size will frequently enter state four, which causes great dissatisfaction. To solve such a mismatch, we further introduce an OS-level power throttling policy that can effectively throttle the peak power $P>P_{F C_{-} \text {Max }}$ by a level of $\alpha \%$, as shown in Figure 5 . Notes that when $P \leq P_{F C_{-} \text {Max }}$, it will not be throttled.

First, we need to estimate the peak power of mobile devices, which is the total power consumption of the major components, including CPU, GPU, screen, WiFi/cellular, camera, bluetooth, and GPS $[6,36,58]$. The CPU, GPU, screen, and WiFi/cellular are the most frequently used, while others, e.g., GPS (even when using application like Google Map, the GPS is connected intermittently rather than continuously), are infrequently used. CPU power $P_{c p u}$ consists of uncore and core 


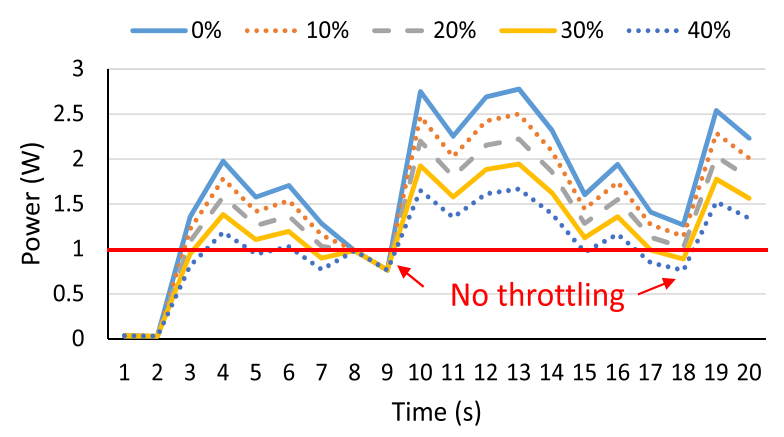

Fig. 5. The illustration of different peak throttling levels.

power consumptions, so we have $P_{c p u}=P_{\text {uncore }}+P_{\text {core }}$ [7]. The core power $P_{\text {core }}$ can be further expressed as the total power of all active cores and idle cores, thus, $P_{\text {core }}=\sum P_{i}$ [20]. For idle cores, their power can be approximated by constants depending on their state [63]. For active cores, their power consumption can be estimated by $P_{\text {dynamic }}+P_{\text {static }}$, and the dynamic power can be further estimated by $P_{\text {dynamic }}=\alpha * C V^{2} f$, where $\alpha$ is a constant, $C$ is the capacitance, $V$ is the voltage, and $f$ is the frequency $[28,41]$. The voltage scales linearly with the frequency under different granularity, i.e., $V_{i}=\theta_{1} * f+\theta_{2}[23,29]$. If per-core voltage scaling is supported, then $f$ is the core frequency. If per-cluster voltage scaling is adopted, then $f$ is the maximal frequency in the cluster. Similarly, the GPU power model can follow the same methodology as multi-core CPU, except state-of-the-art GPUs only support per-chip voltage and frequency scaling [35, 52]. The screen power can be modeled by the multiplication of a quadratic function of the brightness and a linear combination of the average red, green, and blue color intensities of all pixels [58]. The WiFi/cellular power is simply a linear function to the transmission rate $D$ [58]. The powers of CPU, GPU, screen, and WiFi/cellular can be controlled by setting proper frequency (for CPU and GPU), brightness (for screen), and maximal connection speed (for WiFi/cellular) in the OS. For the infrequent used ones, their powers can be simply modeled by some constant values if they are on $[36,58]$.

With peak throttling level $\alpha \%$, the power of each major running components in the device is capped by $1-\alpha \%$. The power of screen and WiFi module can be reduced by adjusting the brightness $L$ and the transmission rate $D$, respectively. The power of GPU can be reduced by adjusting the chip frequency and voltage together. For CPU with per-core frequency and voltage scaling capability, to achieve the overall power cap, the frequency and voltage of each active core will be reduced by a percentage calculated by $\alpha * P_{c p u} / P_{\text {active }}$, unless the lowest possible frequency is reached for some cores. If it happens, then the frequency and voltage of the core with the highest frequency will be further reduced until overall power cap is met.

\subsection{Bridging the Ratio of FC/Battery and Peak Throttling Level}

We first denote $\eta \%$ as the percentage of size allocated for the battery in the hybrid system. Then, the percentage for FC will be $1-\eta \%$. If equipped on mobile devices, then a hybrid system with certain $\mathrm{FC} /$ battery ratio should be coupled with corresponding $\alpha \%$ peak throttling level power management policy to avoid frequently enter state four. In this section, we quantitatively study their relationship to enable the hybrid system for mobile devices. Clearly, with a large throttling level, high power demand is reduced and small battery size in the hybrid system suffices. Therefore, there is a one-to-one mapping between them, and we define the pair of $\langle\alpha \%, \eta \%\rangle$ as the configuration of our hybrid system, where $\alpha \%$ specifies the peak throttling level and $\eta \%$ indicates the portion of battery in the hybrid system. 


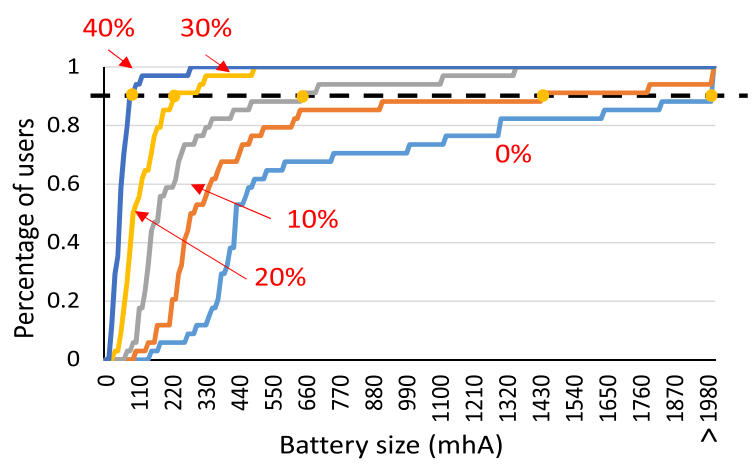

Fig. 6. The percentage of the users whose power requirements are served given battery capacities under different peak throttling levels.

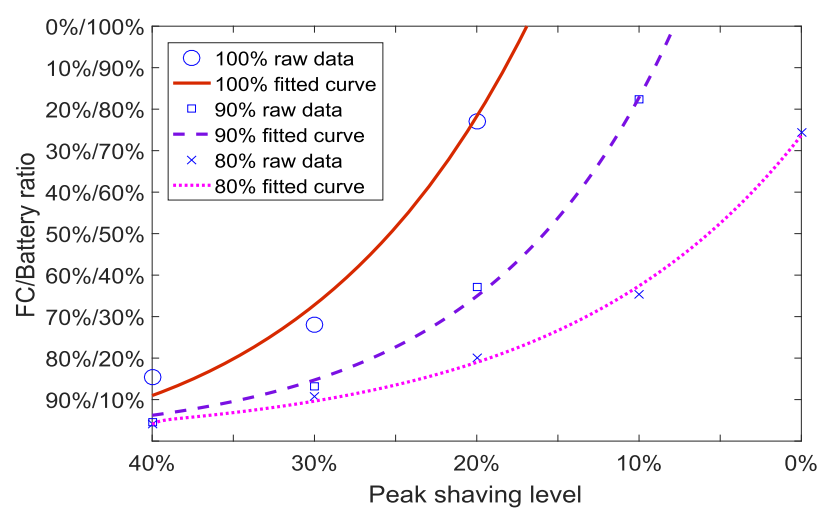

Fig. 7. FC/Battery ratio with different peak throttling levels. Different curves correspond to different coverages.

As discussed in Section 2.3, mobile users have highly diversified power demands. The percentage of users whose power requirements can be satisfied (i.e., the hybrid system never enters state 4) given different battery capacities under $10 \%, 20 \%, 30 \%$, and $40 \%$ peak throttling levels is plotted in Figure 6. The battery is simulated by a classic model Dualfoil, which models the diffusion processes inside the battery and achieves very high accuracy [55]. The LTO battery's specific energy is roughly $1 / 2$ of the LCO battery, and thus the maximum LTO battery capacity is $1750 \mathrm{mAh}$. However, with $0 \%$ or even $10 \%$ peak throttling, it is impossible to satisfy all the users even with a 1980-mAh LTO battery. With 20\%, 30\%, and 40\% peak throttling, 1350-, 490-, and 270-mAh batteries are needed, respectively. If the criteria of satisfying $100 \%$ of the users, i.e., $100 \%$ coverage, is relaxed to $90 \%$, then $0 \%$ is still impossible. We can get the required battery size as $1440,650,230$, and $80 \mathrm{mAh}$ when applying the $10 \%, 20 \%, 30 \%$, and $40 \%$ peak throttling, respectively.

Figure 7 further displays the data pairs of FC/battery ratio and its corresponding peak throttling level and fitted curves to maintain $100 \%, 90 \%$, and $80 \%$ coverages. The first curve is for $100 \%$ user coverage, and it can be observed that at least an $18 \%$ peak throttling technique has to be adopted. To cover $90 \%$ of users (the second curve), at least $8 \%$ peak throttling is needed. If further relaxing to cover $80 \%$ (the third curve) of users, then $0 \%$ peak throttling can be allowed. Given a coverage, FC/battery ratio generally follows exponential relationship with peak throttling level. Thus, we 
introduce the following exponential model:

$$
\eta=d_{1} * e^{(1-c * \alpha)}+d_{2},
$$

where $c, d_{1}$, and $d_{2}$ are parameters. The curve also becomes steeper with higher user coverage, e.g., the curve for $90 \%$ user coverage is steeper than the one for $80 \%$ user coverage. Overall, given a coverage level (e.g., 90\%), there is a set of available configurations, for example, $<10 \%, 82 \%\rangle$, $<20 \%, 37 \%>,<30 \%, 13 \%>,<40 \%, 5 \%>$.

\section{CUSTOMIZING THE HYBRID SYSTEM FOR INDIVIDUAL MOBILE USERS}

Since mobile users have highly diversified demands, a hybrid system with fixed configuration can hardly satisfy everyone. In this section, we build a user satisfaction model, and identify that user satisfaction is strongly affected by the personality. Finally, we explore the customized configuration of a hybrid FC/battery system for individual users based on his/her personality to deliver optimal user satisfaction.

\subsection{The Tradeoff between QoS and Operation Time}

We define the quadruple of CPU frequency, GPU frequency, screen brightness, and Internet connection speed as the Quality-of-Service (QoS). Without explicit mentioning, the QoS degradation or operation time extension are compared against the baseline case, i.e., no peak throttling plus LCO battery. Considering a hybrid system with configuration $\langle\alpha \%, \eta \%\rangle$, the battery percentage in the hybrid system $\eta \%$ can translate to operation time extension $t$ by the following formula:

$$
t=f_{1} *(1-\eta)+f_{2} * \eta
$$

where $f_{1}$ and $f_{2}$ are the ratios of specific energy of FC and LTO battery to the baseline LCO battery, respectively. In our hybrid system, we have $f_{1}=6$ and $f_{2}=0.5$. Meanwhile, a peak throttling level of $\alpha \%$ causes a QoS degradation of $\alpha \%$. With Equation (2), we can quantitatively evaluate the tradeoff between QoS and operation time.

Obviously, adopting a fixed configuration can hardly satisfy every user. Some users may be satisfied with trading more QoS for a longer operation time, while some may be quite sensitive to the QoS degradation and even willing to tolerate the short operation time. In the next section, we build user satisfaction model and identify the best configuration for individual users.

\subsection{Modeling User Satisfaction}

To evaluate users' satisfaction and find the best configuration for them, we conduct a survey on 60 users with half male and half female. Their average age is 25.5 years old, with the youngest 17 years old and the oldest 49 years old. These users are invited to test four peak throttling levels, $10 \%$, $20 \%, 30 \%$, and $40 \%$, with the 10 most popular applications as mentioned before, including Gmail, Google Map, Youtube, Facebook, Google, Google+, Google Text-to-speech, WhatsApp, Google Play Books, and Messenger, on a Samsung Galaxy S9 smartphone [4]. Here we set the peak throttling granularity as $10 \%$, which is good enough for our modeling purpose. Each participant's satisfaction score with the QoS, ranging from 1 (lowest satisfaction) to 10 (higest satisfaction), is recorded for each peak throttling level. To eliminate the impact of prior experiences with the participants' own devices and make a fair comparison, the satisfaction level for $0 \%$ peak throttling is always set to the highest score 10. In other words, the satisfaction score is a relative value based on the common baseline with no peak throttling. Meantime, using Equation (2), the device operation time extensions correspond to these four configurations are $1.6 \times, 4 \times, 5.3 \times$, and $5.7 \times$, respectively. Users are instructed with these device operation time extensions and their satisfaction scores are recorded as well. Again, to make a fair comparison, the satisfaction level for $1 \times$, i.e., no operation 
time extension, is always set to 1 . Now, for each participant, he/she has two sets of satisfaction scores, with one set for QoS and another for operation time extension. The overall satisfaction score per configuration for certain participant can be acquired by simply adding his/her two scores together, and the optimal configuration for him/her is the one with the highest overall satisfaction score. Normal distribution is widely adopted to model survey scores [22]. Empirically, we find each participant's scores can well fit the normal distribution with respect to the peak throttling level $\alpha \%$. Thus, the total satisfaction score $S_{\text {total }}$ for a group of $n$ users can be modeled as the summation of each user's satisfaction score $S_{i}$, i.e.,

$$
S_{\text {total }}=\sum_{i=1}^{n} S_{i}, \quad \text { where } S_{i}=\frac{1}{\sqrt{2 \pi \sigma^{2}}} e^{-\frac{\left(\alpha_{i}-\alpha_{i}^{\prime}\right)^{2}}{2 \sigma^{2}}} .
$$

In this formula, $\alpha_{i}$ and $\alpha_{i}^{\prime}$ are the real and optimal peak throttling levels, respectively. Clearly, to optimize $s$, we simply need to set $\alpha_{i}=\alpha_{i}^{\prime}$ for each $i$. Then, the next question becomes how to find the optimal peak throttling level for each user. As our key observation, we find that the mobile user's personality has great impact on his or her preference with QoS and operation time. Leveraging this observation, we can customize the optimal hybrid FC and battery system for each individual user.

\subsection{Predicting Optimal Peak Throttling}

Personality refers to individuals' characteristic patterns of thought, emotion, and behavior, together with the psychological mechanisms-hidden or not-behind those patterns [19]. To describe someone's personality, Big-Five traits are widely used, which include Extroversion (E), Agreeableness (A), Conscientiousness (C), Neuroticism (N), and Openness to Experience (O) [34]. Extrovert people are usually recognized as active, assertive, energetic, enthusiastic, outgoing, talkative, and so on. Agreeable people are often viewed as more appreciative, forgiving, generous, kind, and sympathetic. Conscientious people tend to be organized and have a strong sense of duty. They are normally described as efficient, organized, planful, reliable, responsible, and thorough. Neurotic people are typically anxious, self-pitying, tense, touchy, unstable, and full of worry. People who are open to experiences are commonly artistic, curious, imaginative, insightful, and original and have wide interests.

In the past, many tests have been proposed to evaluate the Big-Five personality traits. For example, the Coopersmith Self-Esteem Inventory (SEI) [9] contains 25 items and the NEO-PersonalityInventory (NEO-FFI) [11] includes 60 items. Recently, a brief Ten Item Personality Inventory (TIPI) has been proposed by Gosling et al. [47], which significantly reduces the effort required to complete the personality test. The 10 items in the TIPI are as follows: (1) I am reserved, (2) I am generally trusting, (3) I tend to be lazy, (4) I am relaxed, handle stress well, (5) I have few artistic interests, (6) I am outgoing, sociable, (7) I tend to find fault with others, (8) I do a thorough job, (9) I get nervous easily, and (10) I have an active imagination. Each of these 10 items will be answered by the participants with a scale from 1 (strongly disagree) to 7 (strongly agree). With all the scales for the 10 items, the personality scores could be calculated based on the model described in Reference [47]. For each of the five personality traits, its score also ranges from 1 to 7 .

We also let the test users, as mentioned in Section 4.2, take TIPI questionnaires, and their personality scores (i.e., the scores of the Big-Five traits) are calculated by the model from Reference [47]. The mean personality scores of the survey participants are E:3.8, A:4.4, C:5, N:4.5, O:4.6 with the standard variation of E:1.7, A:1.6, C:1.4, N:1.5, O:1.1. After analyzing the personality and users' optimal peak throttling levels, we observe a strong correlation. We identify that the conscientiousness score is negatively related with the peak throttling level, which means conscientious people prefer high QoS over long operation time. The reason is that this type of user is highly 


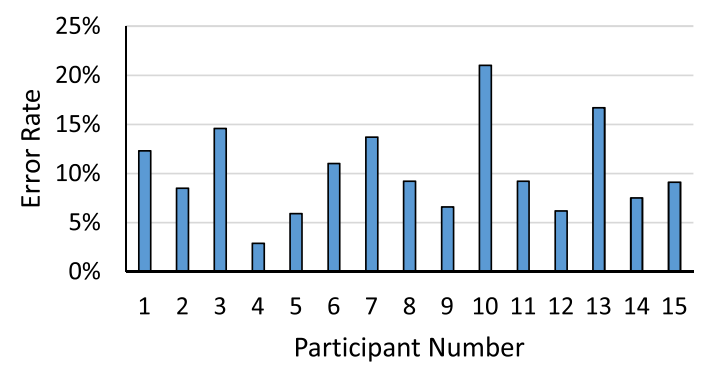

Fig. 8. The prediction accuracy for all participants in the testing set.

organized and usually has a regular charging behavior, and, thus, the long operation time is less attractive to them. The Neuroticism score is positively related with the peak throttling level, because it has been found that neurotic people generally use smartphones less often, and, thus, they prefer long operation time rather than high QoS. For people with high Openness-to-Experience and Extroversion scores, they tend to choose peak throttling level between $20 \%$ to $30 \%$, because both QoS and the operation time matter to them.

Finally, we build a linear regression model to predict users' optimal peak throttling level based on their personality. We denote the personality scores for all survey participants as $\mathbf{P}$. The optimal peak throttling levels of all participants are denoted as $\hat{\alpha}^{\prime}$. We randomly split the data with $80 \%$ for training set and $20 \%$ for testing set. The parameter $\beta$ can be acquired by minimizing the means square error as follows:

$$
\min _{\beta}\left\|\hat{\alpha}^{\prime}-\mathbf{P} * \beta\right\|^{2}
$$

With new data $p$ (i.e., personality score), his/her optimal peak throttling level can be predicted by $p * \beta$. The prediction accuracy for all participants in the testing set is shown in Figure 8 , and the average accuracy is $>89 \%$.

\section{EVALUATION}

\subsection{Experiment Setup}

We build a prototype to evaluate the hybrid FC and battery system. For the FC subsystem, we use the Horizon hydrogen FC charger to simulate the DMFC, since the commercial DMFC is not available for the smartphone yet and the methanol and hydrogen based FCs have similar characteristics [46]. The Horizon FC charger uses USB output, which is $5 \mathrm{~V}$ by its standard, and, thus, the design of the prototype is slightly different from Figure 4. We use a Texas Instrument (TI) TPS22944 0.2-A current limit load switch (its input voltage ranges from $1.62 \mathrm{~V}$ to $5.5 \mathrm{~V}$ ), which can limit the FC output power to $1 \mathrm{~W}$. Since the device we use requires a $5-\mathrm{V}$ input, the output voltage of the FC does not need to be further regulated. We use the TI TPS63024 buck/boost converter, which can convert the $5-\mathrm{V}$ output of the FC to $2.9 \mathrm{~V}$ (the charging voltage of the LTO battery), as the DC-DC converter for the battery charging. For the battery, we use a 1,500-mAh LTO battery managed by the TI BQ27532EVM battery charging solution evaluation module, which can charge the battery and also provide the battery status, including remaining capacity, and so on, to the EMS via the TI EV2300 interface board. The output power of the battery is only $2.4 \mathrm{~V}$, which is converted to 5 $\mathrm{V}$ by the TI TPS61230EVM boost converter. The converters can be controlled by enabling signals, and, thus, there is no need for extra switches. The voltage and current sensors are LTC2990. We implement the EMS based on the micro-controller TI MSP-EXP430.

We use the Odroid XU3 ARM development board as the device whose configuration is on par with the state-of-the-art smartphones. The CPU of the board is the Exynos5422 SoC, which 


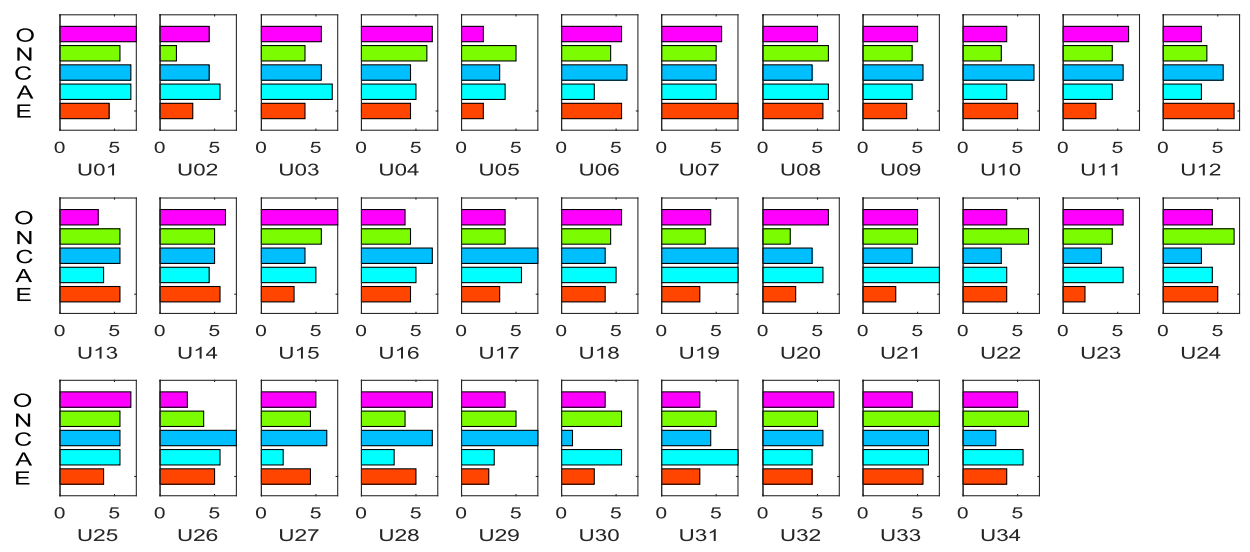

Fig. 9. The personality scores (i.e., the score for each personality trait) for all test users.

contains one out-of-order quad-core Cortex-A15 cluster and one in-oder quad-core Cortex-A7 cluster (ARM BIG.little architecture). The GPU is Mali-T628 GPU, and the main memory is 2G LPDDR3 RAM. It contains built-in power monitors for CPU, GPU, and memory. The Odroid XU3 board is cooled by a fan, which is powered separately in our platform, since they are usually not available for smartphones. Also, we connect the ARM board with a 5 -in. multi-touch screen and a WiFi module; their power are measured by REGOL DM3058 multi-meter separately.

We compare five fixed configurations, including baseline (no peak throttling plus a 3,500-mAh LCO battery), C1 (<10\%, $82 \%>$, i.e., $10 \%$ throttling plus $82 \%$ LTO battery), C2 $(<20 \%, 37 \%>)$, C3 $(<30 \%, 13 \%>)$, and $\mathrm{C} 4(<40 \%, 5 \%>)$, with a personality-aware configuration, which selects the optimal one of these five for each individual user based on his/her personality.

To evaluate our proposed technique, we recruited another 34 test users, who are different from the previous survey participants. We recorded their smartphone usage, i.e., when and which application they are using, for 3 weeks. We did not record the actions, e.g., touch, swipe, and so on, since replaying these actions in a different platform is impractical due to system configuration and software version variation. Instead, we create a base benchmark for each application, e.g., Facebook, in our experiment platform. We first run each base benchmark for a sufficiently long time with the default interactive governor on the ARM board. Meanwhile, we use the HiroMacro auto-touch to record the actions, e.g., touch, swipe, and so on, of a specific test user using our experiment platform. We also use the Linux perf tool to record the progress of execution by periodically dumpling the instruction count. Then, we can link the user actions to the instruction count rather than time, since the time can change with different frequencies. The base benchmark is used to replace an execution trace of corresponding application. For example, as user "U1" uses Facebook for 10,000 instructions (under default interactive governor), we can simple cut 10,000 instructions from our base benchmark use it for "U1." In this way, we can re-generate the execution traces for all the users.

\subsection{Personality Scores and Optimal Peak Throttling Levels}

The personality scores for all the test users are shown in Figure 9. ${ }^{1}$ As shown, these users have different scores on the five traits. For instance, "U07" exhibits quite strong tend to the extroversion trait, while "U26" leans more toward the conscientiousness trait. The predicted optimal throttling

\footnotetext{
${ }^{1}$ Our test users have agreed on the disclosure of their personality and preference data.
} 
Table 1. The Predicted Optimal Peak Throttling Levels for All the Test Users

\begin{tabular}{llllllll}
\hline UID & Opt & UID & Opt & UID & Opt & UID & Opt \\
\hline U01 & $20 \%$ & U10 & $10 \%$ & U19 & $0 \%$ & U28 & $20 \%$ \\
U02 & $10 \%$ & U11 & $20 \%$ & U20 & $10 \%$ & U29 & $10 \%$ \\
U03 & $20 \%$ & U12 & $20 \%$ & U21 & $20 \%$ & U30 & $40 \%$ \\
U04 & $40 \%$ & U13 & $30 \%$ & U22 & $40 \%$ & U31 & $30 \%$ \\
U05 & $20 \%$ & U14 & $30 \%$ & U23 & $30 \%$ & U32 & $30 \%$ \\
U06 & $20 \%$ & U15 & $30 \%$ & U24 & $40 \%$ & U33 & $40 \%$ \\
U07 & $30 \%$ & U16 & $20 \%$ & U25 & $30 \%$ & U34 & $30 \%$ \\
U08 & $40 \%$ & U17 & $10 \%$ & U26 & $0 \%$ & & \\
U09 & $20 \%$ & U18 & $30 \%$ & U27 & $20 \%$ & & \\
\hline
\end{tabular}

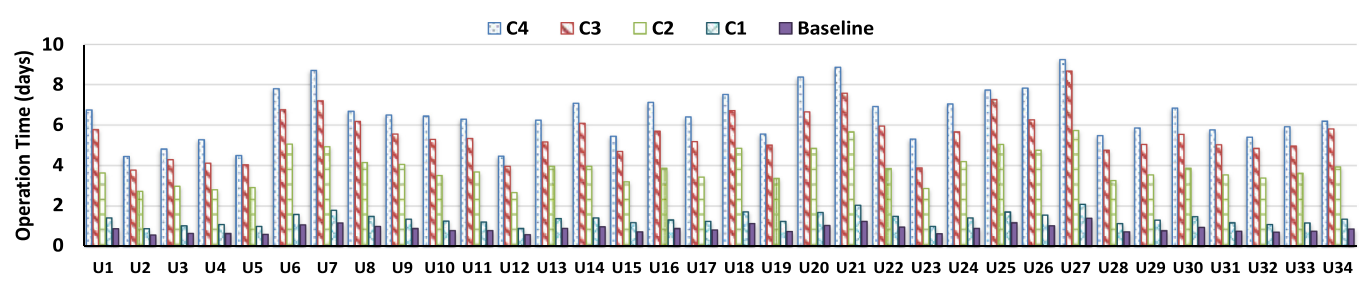

Fig. 10. The operation time in days for all the test users with different configurations.

levels for test users are shown in Table 1. As shown in the table, some users may prefer high QoS (i.e., $0 \%$ peak throttling), while others desire longer operation time (i.e., $40 \%$ peak throttling). Obviously, any fixed configuration can only fully satisfy a small group of users, and partially satisfy or even completely dissatisfy others. While setting the optimal peak throttling level for each individual user can always fully satisfy all the users, and, thus, is better than any fixed configurations.

\subsection{Results}

In this study, we evaluate three most important metrics for the hybrid system: the operation time, the battery lifecycle, and the user satisfaction score.

The operation time for each test user is shown in Figure 10, and the average results are shown in Figure 13(a). Since the optimal peak throttling level per user is already listed in Table 1, the operation time under a personality-aware optimal configuration is equal to one of the throttling levels from $0 \%$ to $40 \%$. Therefore, Figure 10 does not show its operation time to avoid the redundant result presentation. For the baseline LCO battery, the averaged operation time is slightly less than 1 day, and it can be extended to 1.3 days with $10 \%$ peak throttling. With $20 \%, 30 \%$, and $40 \%$ peak throttling, as the percentage of the FCs gets larger, the averaged operation time is further improved to 3.9, 5.6, and 6.5 days. The personality-aware peak throttling can power the device for 4.3 days on average across all users. For some users with light usage, e.g., "U21" and "U27," the baseline LCO battery can last for 1.24 and 1.39 days, respectively. With $40 \%$ peak throttling, their operation time can be even extended to 8.86 and 9.25 days, respectively. For some users with heavy usage, e.g., "U02" and "U03," the baseline case can only last for around 0.6 days, and the hybrid system with $40 \%$ peak throttling can last more than 4 days.

The battery lifecycle is also an important metric, because battery aging is a significant problem, and it needs to be frequently charge/discharged in our hybrid system. The battery lifecycle for each user is shown in Figure 11, and the average is shown in Figure 13(b). Similarly, Figure 11 


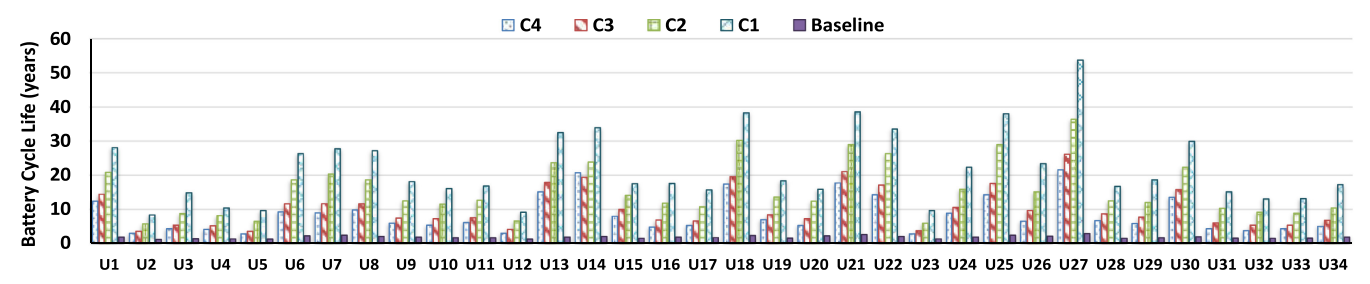

Fig. 11. The battery lifecycle in years for all the test users with different configurations.

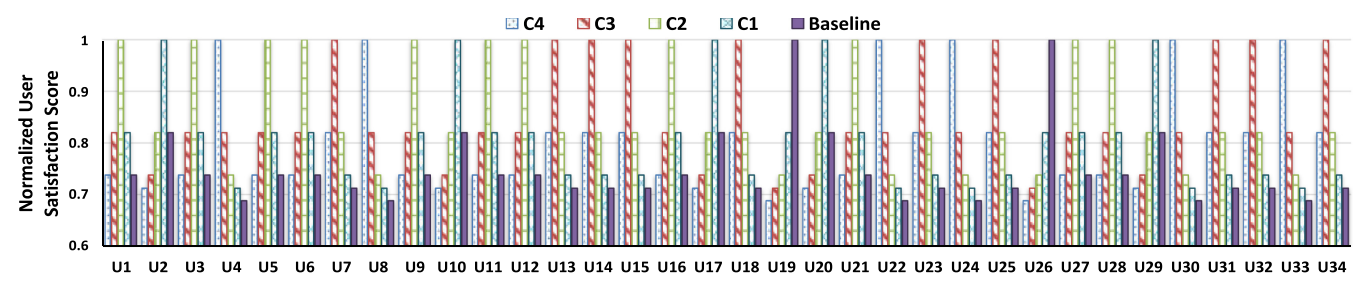

Fig. 12. The normalized user satisfaction score for all test users with different configurations (normalized to the highest score).

does not present the battery lifecycle under the personality-aware optimal configuration to avoid showing the redundant result. Excitingly, the LTO battery in the hybrid system can endure much longer than the LCO battery. Even with $40 \%$ peak throttling that uses the smallest LTO battery requiring quite frequent charging/discharging, the LTO battery can still last for around 8.4 years compared with only 1.8 years of the LCO battery. With $10 \%$ peak throttling, the LTO battery can last for more than 20 years on average. For some user, like "U27," the LTO battery can last for 50 years. Since the FC does not have the same significant aging problem as the battery, the hybrid system is expected to last much longer than the LCO battery [46].

The satisfaction score normalized to the highest one for each user is shown in Figure 12, and the average is shown in Figure 13(c). Again, the score under the personality-aware technique is not shown in Figure 12 to avoid the redundant result presentation. The baseline case has the lowest score 0.75 . The optimal has the highest satisfaction score of 1 , and the second optimal is the $20 \%$ peak throttling with a satisfaction score of $\sim 0.85$.

Overall, our personality aware optimal configuration can achieve $4 \times$ longer operation time, $6 \times$ longer battery lifecycle, and 25\% higher user satisfaction score compared with the baseline LCO battery. C2 $(<20 \%, 37 \%>)$ achieves the higher QoS score than other fixed configurations. Our optimal configuration can achieve $11 \%$ improvement on operation time extension, $15 \%$ higher user satisfaction score comparing with $\mathrm{C} 2$. Although the battery lifecycle of optimal configuration is $20 \%$ lower than C2, 12.5 years of battery lifecycle is more than enough for normal users, and the disadvantage of 3 years' shorter battery lifecycle is negligible.

Finally, to analyze the cost of the hybrid system, we consider the cost of the fuel (methanol), the cell (e.g., the anode, the cathode), and the LTO battery. The cost of the methanol is negligible, and the cost of cell itself is around $\$ 2$ for the size required by smartphones [8]. The cost of the LTO battery is about $\$ 2.5 / \mathrm{Ah}$ compared to $\$ 1.5 / \mathrm{Ah}$ for the LCO battery. Thus, for the personality-aware optimal configuration, the overall cost is only about half of the LCO battery. Here we only consider the cost of some basic components, which is scaled from the existing products. In real world, the cost is highly related with some other factors, e.g., the maturity of technology, which is beyond the discussion of this article. 


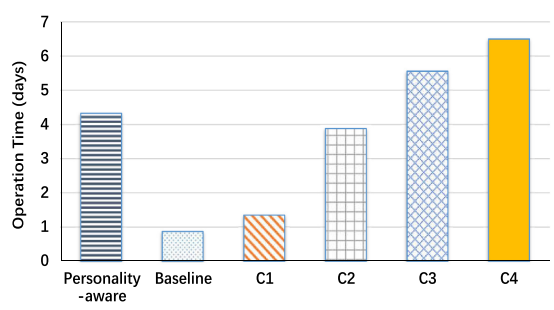

(a)

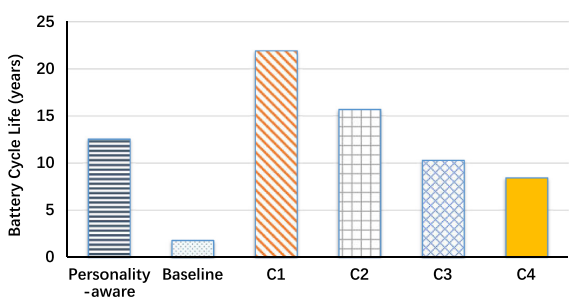

(b)

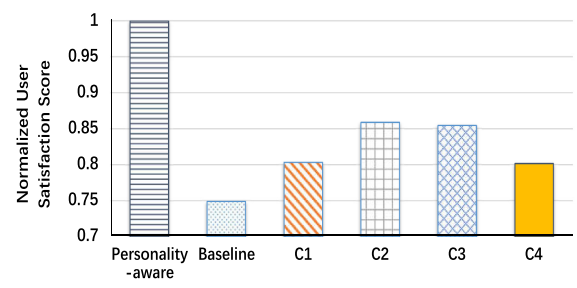

(c)

Fig. 13. The average (a) operation time, (b) battery lifecycle, and (c) user satisfaction score with different configurations.

\section{RELATED WORK}

In the past, there were many work using the DMFC for the portable devices $[1,2,12,14,43,54]$. Some prototypes of the passive air-breathing DMFC have been built for mobile devices $[1,14,54]$. C. Chen et al. identified that the passive air-breathing DMFC's low specific power is highly related to the fabrication conditions and further explored proper manufacturing process by adjusting its physical parameters $[12,13]$. In addition, a more efficient catalyst has been proposed to boost the performance of the DMFC [43]. K. Lee et al. have designed a fuel cell and battery hybrid system; however, the usage characteristics of mobile devices are not considered [31]. Also, many studies have been conducted to introduce the personality into the mobile computing. S. Walsh et al. investigated the impact of the personality on smartphone usage for Australian youth [56]. The personality also has been found to be highly related with the usage of games and the Internet $[24,45]$. However, smartphone usage can be used to infer the users' personality and even stress level $[21,40]$. K. Yan et al. have re-defined the QoS definition for mobile devices and explored the optimal management policy based on users' personalities [60].

There have been many studies on the mobile architecture and system. Some studies have been performed to analyze and model the energy consumption of mobile devices [6, 36, 58]. Y. Zhu et al. proposed the event-based scheduling strategy to optimize the energy-efficient QoS. The accelerators (or IP cores) in the mobile devices also attracted a lot of attention recently. A domain-aware coordinated DVFS policies, which can coordinate the DVFS state for each component of the mobile devices, was proposed to achieve high energy efficiency for the frame-based applications [37]. A new IP virtualization framework (VIP) was proposed for mobile devices, which allows many IPs to be chained together and be virtualized as a single one [38]. DORA, a dynamic frequency controller for energy efficient mobile web browsing was introduced [49]. For streaming-based mobile applications, a combination of race-to-sleep, content, and display caching techniques was proposed to improve energy efficiency [61]. Human factors were also considered for architecture design and power management. Some hardware, e.g. eye tracker, force sensor, and so on, have been used to collect human physiological traits, which guide the control of CPU frequency [51]. Further, the link 
between micro-architecture level measurement and user satisfaction was explored, which does not rely on external hardware [50]. However, none of these works targets fuel cell and a battery hybrid system for mobile devices or considers user personality.

\section{CONCLUSION}

In this study, we develop a hybrid FC and battery system as the power supply for the mobile devices to significantly extend the device operation time, while still satisfying users' diversified power demands. The hybrid system uses passive air-breathing DMFC and an LTO battery, which achieves both high specific energy and specific power. We examine real-world smartphone usage data, LiveLab, and find that a naive hybrid power system cannot satisfy many users' high power demands. We then propose an OS-level power management policy, which reduces the device power consumption by $\alpha \%$ for each power peak to solve the mismatch between the power supply and power demands. This technique trades the QoS for a large FC size, thus much longer device operation time. Furthermore, we observe that user personality largely affects his/her satisfaction with the QoS loss and the operation time extension. Thus, applying a hybrid system with fixed configuration (i.e., peak throttling level coupled with corresponding FC/battery ratio) is unable to satisfy each user. We then explore customized hybrid FC and battery system configuration for each individual user according to his/her personality, which achieves the optimal user satisfaction per user. The experimental results show that our personality-aware hybrid FC and battery solution can achieve $4 \times$ longer operation time and $25 \%$ higher satisfaction score comparing with the baseline LCO battery.

\section{REFERENCES}

[1] M. Philosoph N. Rudoy E. Peled A. Blum, and T. Duvdevani. 2003. Water-neutral micro direct-methanol fuel cell (DMFC) for portable applications. 7. Power Sources 117, 1-2 (May 2003), 22-25.

[2] R. K. Nema, A. Kirubakaran, and Shailendra Jain. 2009. A review on fuel cell technologies and power electronic interface. Renew. Sust. Energy Rev. 13, 9 (Dec. 2009), 2430-2440.

[3] Marshall Miller and Andrew Burke. 2009. Performance characteristics of lithium-ion batteries of various chemistries for plug-in hybrid vehicles. In Proceedings of the International Battery, Hybrid and Fuel Cell Electric Vehicle Symposium.

[4] AndroidRank. 2015. Retrieved from http://www.androidrank.org/.

[5] Debangshu Das, Arnab Adhikari, Arnab Kundu, and Rajiv Kumar Utsav. 2014. A comparative analysis of optimised lithium-ion battery model and its application. Int. f. Emerg. Technol. Adv. Eng. 4, 4 (2014).

[6] Aaron Carroll and Gernot Heiser. 2010. An analysis of power consumption in a smartphone. In Proceedings of the 2010 USENIX Conference on USENIX Annual Technical Conference (USENIXATC'10). USENIX Association, 21-21. http://dl.acm.org/citation.cfm?id=1855840.1855861

[7] A. Carroll and G. Heiser. 2014. Unifying DVFS and offlining in mobile multicores. In Proceedings of the 2014 IEEE 19th Real-Time and Embedded Technology and Applications Symposium (RTAS'14). 287-296. DOI : https://doi.org/10.1109/ RTAS.2014.6926010

[8] Jacob S. Spendelow, John Kopasz, David Peterson, Nancy L. Garland, Donna Lee, Ho Jason, Marcinkoski Kathi, Epping Martin, Reginald Tyler, Cassidy Houchins, Greg J. Kleen, and Dimitrios C. Papageorgopoulos. 2012. U.S. DOE progress towards developing low-cost, high performance, durable polymer electrolyte membranes for fuel cell applications. Membranes 2, 4 (Dec. 2012), 855-878.

[9] Stanley Coopersmith. 1968. The Antecedents of Self-Esteem (A Series of Books in Behavioral Science). W. H. Freeman and Company.

[10] Horacio R. Corti and Ernesto R. Gonzalez. 2014. Direct Alcohol Fuel Cells: Materials, Performance, Durability and Applications. Springer.

[11] Paul Costa and Robert McCrae. 1995. Domains and facets: Hierarchical personality assessment using the revised NEO personality inventory. F. Res. Pers. 64, 1 (Feb 1995), 21-50.

[12] P. Yang and C. Y. Chen. 2003. Performance of an air-breathing direct methanol fuel cell. fournal of Power Sources 141, 1 (Feb 2005), 24-29.

[13] Y. S. Lee, K. F. Lin, C. Y. Chen, and P. Yang. 2005. Fabrication of electrocatalyst layers for direct methanol fuel cells. f. Power Sources 141, 1 (Feb 2005), 24-29. 
[14] Daejin Kim, Eun Ae Cho, Seong-Ahn Hong, Seong-Ahn Oh, and Heung Yong Ha. 2004. Recent progress in passive direct methanol fuel cells at KIST. Fournal of Power Sources 130, 1 (2004), 172-177. DOI : 10.1016/j.jpowsour.2003.12.023

[15] Jessica Dolcourt. 2015. Retrieved from http://www.cnet.com/news/smartphones-with-long-battery-life-roundup/l.

[16] M. Dudek, Piotr Tomczyk, Piotr Wygonik, Mariusz Korkosz, Piotr Bogusz, and Bartlomiej Lis. 2013. Hybrid fuel cell - battery system as a main power unit for small unmanned aerial vehicles (UAV). Int. f. Electrochem. Sci. 8, 6 (2013), 8442-8463.

[17] Hossein Falaki, Ratul Mahajan, Srikanth Kandula, Dimitrios Lymberopoulos, Ramesh Govindan, and Deborah Estrin. 2010. Diversity in mobile devices usage. In Proceedings of the 8th International Conference on Mobile Systems, Applications, and Services (MobiSys'10). ACM, New York, NY, 179-194. DOI : https://doi.org/10.1145/1814433.1814453

[18] N. Femia, G. Petrone, G. Spagnuolo, and M. Vitelli. 2005. Optimization of perturb and observe maximum power point tracking method. IEEE Trans. Power Electr. 20, 4 (Jul. 2005), 963-973. DOI : https://doi.org/10.1109/TPEL.2005.850975

[19] David C. Funder. 2010. The Personality Puzzle (5th ed.). W. W. Norton \& Company.

[20] B. Goel and S. A. McKee. 2016. A methodology for modeling dynamic and static power consumption for multicore processors. In Proceedings of the 2016 IEEE International Parallel and Distributed Processing Symposium (IPDPS'16) 273-282. DOI : https://doi.org/10.1109/IPDPS.2016.118

[21] Gokul Chittaranjan, Jan Blom, and Daniel Gatica-Perez. 2013. Mining large-scale smartphone data for personality studies. Personal Ubiquitous Comput 17, 3 (March 2013), 433-450. DOI : https://doi.org/10.1007/s00779-011-0490-1

[22] Sue Gordon. 2016. The Normal Distribution. Ph.D. Dissertation. University of Sydney.

[23] Ujjwal Gupta. 2018. Power-Performance Modeling and Adaptive Management of Heterogeneous Mobile Platforms. Ph.D. Dissertation. Arizona State University.

[24] Sarah Butt, James G. Phillips, and Alex Blaszczynski. 2007. Personality and self-reported use of mobile phones for games. Cyber Psychol. Behav. 9, 6 (2007), 753-758.

[25] S. Kamarudin, F. Achmad, and W. Dauda. 2009. Overview on the application of direct methanol fuel cell (DMFC) for portable electronic devices. Int. F. Hydr. Energy 34, 16 (2009), 6902-6916.

[26] N. Karami, R. Outbib, and N. Moubayed. 2012. Fuel flow control of a PEM fuel cell with MPPT. In Proceedings of the 2012 IEEE International Symposium on Intelligent Control. 289-294. DOI : https://doi.org/10.1109/ISIC.2012.6398246

[27] E. G. Karvelas, D. G. Koubogiannis, A. Hatziapostolou, and I. E. Sarris. 2016. The effect of anode bed geometry on the hydraulic behaviour of PEM fuel cells. Renew. Energy 93 (2016), 269-279. https://www.sciencedirect.com/science/ article/abs/pii/S0960148116301823.

[28] Stefanos Kaxiras and Margaret Martonosi. 2008. Computer Architecture Techniques for Power-Efficiency (1st ed.). Morgan \& Claypool Publishers.

[29] Wonyoung Kim, M. S. Gupta, G. Wei, and D. Brooks. 2008. System level analysis of fast, per-core DVFS using on-chip switching regulators. In Proceedings of the 2008 IEEE 14th International Symposium on High Performance Computer Architecture. 123-134. DOI : https://doi.org/10.1109/HPCA.2008.4658633

[30] U. Krewer, Y. Song, Kai Sundmacher, V. John, R. Lübke, Gunar Matthies, and Lutz Tobiska. 2004. Direct methanol fuel cell (DMFC): Analysis of residence time behaviour of anodic flow bed. Chem. Eng. Sci. 59, 1 (2004), 119-130. DOI : https://doi.org/10.1016/j.ces.2003.09.029

[31] Kyungsoo Lee, Naehyuck Chang, Jianli Zhuo, Chaitali Chakrabarti, Sudheendra Kadri, and Sarma Vrudhula. 2008. A fuel-cell-battery hybrid for portable embedded systems. ACM Trans. Des. Autom. Electron. Syst. 13, 1, Article 19 (Feb. 2008), 34 pages. DOI : https://doi.org/10.1145/1297666.1297685

[32] Hong Liu, Peiwen Li, Daniel Juarez-Robles, Kai Wang, and Abel Hernandez-Guerrero. 2014. Experimental study and comparison of various designs of gas flow fields to PEM fuel cells and cell stack performance. Front. Energy Res. 2, 2 (2014). DOI : https://doi.org/10.3389/fenrg.2014.00002

[33] Longjun Liu, Chao Li, Hongbin Sun, Yang Hu, Juncheng Gu, Tao Li, Jingmin Xin, and Nanning Zheng. 2015. HEB: Deploying and managing hybrid energy buffers for improving datacenter efficiency and economy. In Proceedings of the 42nd Annual International Symposium on Computer Architecture (ISCA'15). ACM, 463-475. DOI : https://doi.org/10. $1145 / 2749469.2750384$

[34] Robert R. McCrae and Oliver P. John. 1992. An introduction to the five-factor model and its applications. journal of personality. F. Pers. 60, 2 (1992), 175-215.

[35] Xinxin Mei, Ling Sing Yung, Kaiyong Zhao, and Xiaowen Chu. 2013. A measurement study of GPU DVFS on energy conservation. In Proceedings of the Workshop on Power-Aware Computing and Systems (HotPower'13). ACM, New York, NY. DOI : https://doi.org/10.1145/2525526.2525852

[36] R. Murmuria, Jeffrey Medsger, A. Stavrou, and J. M. Voas. 2012. Mobile application and device power usage measurements. In Proceedings of the 2012 IEEE Sixth International Conference on Software Security and Reliability (SERE'12). 147-156. DOI : https://doi.org/10.1109/SERE.2012.19 
[37] N. C. Nachiappan, P. Yedlapalli, N. Soundararajan, A. Sivasubramaniam, M. T. Kandemir, R. Iyer, and C. R. Das. 2015. Domain knowledge based energy management in handhelds. In Proceedings of the 2015 IEEE 21st International Symposium on High Performance Computer Architecture (HPCA'15). 150-160. DOI : https://doi.org/10.1109/HPCA.2015. 7056029

[38] Nachiappan Chidambaram Nachiappan, Haibo Zhang, Jihyun Ryoo, Niranjan Soundararajan, Anand Sivasubramaniam, Mahmut T. Kandemir, Ravi Iyer, and Chita R. Das. 2015. VIP: Virtualizing IP chains on handheld platforms. In Proceedings of the 42nd Annual International Symposium on Computer Architecture (ISCA'15). ACM, New York, NY, 655-667. DOI : https://doi.org/10.1145/2749469.2750382

[39] M. Nadal and F. Barbir. 1996. Development of a hybrid fuel cell/battery powered electric vehicle. Int. F. Hydr. Energy 21, 6 (1996), 497-505. DOI : https://doi.org/https://doi.org/10.1016/0360-3199(95)00102-6

[40] Raihana Ferdous, Venet Osmani, and Oscar Mayora-Ibarra. 2015. Smartphone app usage as a predictor of perceived stress levels at workplace. In 9th International Conference on Pervasive Computing Technologies for Healthcare (PervasiveHealth'15). 225-228. DOI : https://doi.org/10.4108/icst.pervasivehealth.2015.260192

[41] Preeti Ranjan Panda, B. V. N. Silpa, Aviral Shrivastava, and Krishnaiah Gummidipudi. 2010. Power-efficient System Design (1st ed.). Springer.

[42] S. Patrabansh, M. Y. El-Sharkh, and M. Alam. 2012. Dynamic modeling of transient response of direct methanol fuel cell. In Proceedings of the 2012 IEEE Industry Applications Society Annual Meeting. 1-12. DOI : https://doi.org/10.1109/ IAS.2012.6374016

[43] Prasad Prakash Patel, Moni Kanchan Datta, Prashanth H. Jampani, Daeho Hong, James A. Poston, Ayyakkannu Manivannan, and Prashant N. Kumta. 2015. High performance and durable nanostructured TiN supported Pt50Ru50 anode catalyst for direct methanol fuel cell (DMFC). fournal of Power Sources 293 (2015), 437-446. DOI : https:// doi.org/10.1016/j.jpowsour.2015.05.051

[44] Thomas Reddy. 2010. Linden's Handbook of Batteries (4th ed.). McGraw-Hill Education.

[45] Richard N. Landers and John W. Lounsbury. 2006. An investigation of Big Five and narrow personality traits in relation to Internet usage. Comput. Hum. Behav. 22, 2 (March 2006), 283-293. DOI : http://dx.doi.org/10.1016/j.chb.2004.06.001

[46] Whitney Colella, Ryan O’Hayre, Suk-Won Cha, and Fritz B. Prinz. 2009. Fuel Cell Fundamentals. John Wiley \& Sons, Inc.

[47] Samuel D. Gosling, Peter J. Rentfrow, and William B. Swann. 2003. A very brief measure of the Big-Five personality domains. Journal of Research in Personality 37, 6 (2003), 504-528. DOI : https://doi.org/10.1016/S0092-6566(03)00046-1

[48] Clayton Shepard, Ahmad Rahmati, Chad Tossell, Lin Zhong, and Phillip Kortum. 2011. LiveLab: Measuring wireless networks and mobile devices users in the field. SIGMETRICS Perform. Eval. Rev. 38, 3 (Jan. 2011), 15-20. DOI: https://doi.org/10.1145/1925019.1925023

[49] D. Shingari, A. Arunkumar, B. Gaudette, S. Vrudhula, and C. Wu. 2018. DORA: Optimizing smartphone energy efficiency and web browser performance under interference. In Proceedings of the 2018 IEEE International Symposium on Performance Analysis of Systems and Software (ISPASS'18). 64-75. DOI : https://doi.org/10.1109/ISPASS.2018.00015

[50] Alex Shye, Berkin Ozisikyilmaz, Arindam Mallik, Gokhan Memik, Peter A. Dinda, Robert P. Dick, and Alok N. Choudhary. 2008. Learning and leveraging the relationship between architecture-level measurements and individual user satisfaction. In Proceedings of the 35th Annual International Symposium on Computer Architecture (ISCA'08). IEEE Computer Society, 427-438. DOI : https://doi.org/10.1109/ISCA.2008.29

[51] A. Shye, Yan Pan, B. Scholbrock, J. S. Miller, G. Memik, P. A. Dinda, and R. P. Dick. 2008. Power to the people: Leveraging human physiological traits to control microprocessor frequency. In Proceedings of the 2008 41st IEEE/ACM International Symposium on Microarchitecture (MICRO-41). 188-199. DOI : https://doi.org/10.1109/MICRO.2008.4771790

[52] K. R. Stokke, H. K. Stensland, P. Halvorsen, and C. Griwodz. 2016. High-precision power modelling of the tegra $\mathrm{k} 1$ variable SMP processor architecture. In Proceedings of the 2016 IEEE 10th International Symposium on Embedded Multicore/Many-core Systems-on-Chip (MCSOC'16). 193-200. DOI : https://doi.org/10.1109/MCSoC.2016.28

[53] Subramanian Sundarrajan, Suleyman I. Allakhverdiev, and Seeram Ramakrishna. 2012. Progress and perspectives in micro direct methanol fuel cell. Int. f. Hydr. Energy 37, 10 (2012), 8765-8786. DOI : https://doi.org/10.1016/j.ijhydene. 2011.12.017 International Conference: Photosynthesis Research for Sustainability.

[54] Takahiro Shimizu, Toshiyuki Momma, Mohamed Mohamedi, Tetsuya Osaka, and Srinivasan Sarangapani. 2004. Design and fabrication of pumpless small direct methanol fuel cells for portable applications. Fournal of Power Sources 137, 2 (2004), 277-283. https://doi.org/10.1016/j.jpowsour.2004.06.008

[55] Marc Doyle, Thomas F. Fuller, and John Newman. 1993. Simulation and optimization of the dual lithium ion insertion cell. F. Electrochem. Soc. 140, 6 (1993), 1526-1533.

[56] Shari P. Walsh, Katherine M. White, and Ross M. Young. 2008. Over-connected? A qualitative exploration of the relationship between Australian youth and their mobile phones. Journal of Adolescence 31, 1 (2008), 77-92. DOI : https:// doi.org/10.1016/j.adolescence.2007.04.004 
[57] Helge Weydahl. 2006. Dynamic Behaviour of Fuel Cells. Ph.D. Dissertation. Norwegian University of Science and Technology.

[58] Fengyuan Xu, Yunxin Liu, Qun Li, and Yongguang Zhang. 2013. V-edge: Fast self-constructive power modeling of smartphones based on battery voltage dynamics. In Proceedings of the 10th USENIX Conference on Networked Systems Design and Implementation (NSDI'13). USENIX Association, Berkeley, CA, 43-56. http://dl.acm.org/citation.cfm?id= 2482626.2482633

[59] Xuan Cheng, Zheng Shi, Nancy Glass, Lu Zhang, Jiujun Zhang, Datong Song, Zhong-Sheng Liu, Haijiang Wang, and Jun Shen. 2007. A review of PEM hydrogen fuel cell contamination: Impacts, mechanisms, and mitigation. Fournal of Power Sources 165, 2 (2007), 739-756. DOI : https://doi.org/10.1016/j.jpowsour.2006.12.012

[60] K. Yan, X. Zhang, J. Tan, and X. Fu. 2016. Redefining QoS and customizing the power management policy to satisfy individual mobile users. In Proceedings of the 2016 49th Annual IEEE/ACM International Symposium on Microarchitecture (MICRO'16). 1-12. DOI : https://doi.org/10.1109/MICRO.2016.7783756

[61] Haibo Zhang, Prasanna Venkatesh Rengasamy, Shulin Zhao, Nachiappan Chidambaram Nachiappan, Anand Sivasubramaniam, Mahmut T. Kandemir, Ravi Iyer, and Chita R. Das. 2017. Race-to-sleep + Content Caching + Display Caching: A recipe for energy-efficient video streaming on handhelds. In Proceedings of the 50th Annual IEEE/ACM International Symposium on Microarchitecture (MICRO'17). ACM, New York, NY, 517-531. DOI: https:// doi.org/10.1145/3123939.3123948

[62] Jiujun Zhang and Hansan Liu. 2010. Electrocatalysis of Direct Methanol Fuel Cells: From Fundamentals to Applications. John Wiley \& Sons, Inc.

[63] Y. Zhang, Y. Liu, Xuanzhe Liu, and Q. Li. 2017. Enabling accurate and efficient modeling-based CPU power estimation for smartphones. In Proceedings of the 2017 IEEE/ACM 25th International Symposium on Quality of Service (IWQoS'17). 1-10. DOI : https://doi.org/10.1109/IWQoS.2017.7969112

Received January 2019; revised May 2019; accepted August 2019 